\title{
Scheduling BTS Power Levels for Green Mobile Computing
}

\author{
Hemalatha M. ${ }^{1}$, Prithiviraj V. ${ }^{2}$ and Jayasri T. ${ }^{3}$ \\ ${ }^{1}$ Department of ECE, Narayana Engineering College, Nellore, \\ Andhra Pradesh, India \\ ${ }^{2}$ Rajalakshmi Institute of Technology, Chennai, Tamil Nadu, India \\ ${ }^{3}$ Tata Consultancy Services, Chennai, Tamil Nadu, India \\ Corresponding Authors: mkhema@yahoo.com; \{profvpraj; \\ jayasri5591\}@gmail.com
}

Received 2 Feb 2015; Accepted 5 March 2015;

Publication 29 May 2015

\begin{abstract}
The world has become sophisticated and man started depending upon various things in a large part of everyday life to enhance daily way of living which includes the most useful gadgets. These resources have made life easier, but there is a lot of concern over the possible health risks due to these radiations. Considering the objectives of Green Mobile Computing, this work proposes to dynamically allocate BTS's transmission power level according to the requirement of number of users in a particular radio cell. The number of users is more during the daytime when compared to night time, so the power level can be dynamically reduced to overcome the hazardous effects of radiation. A scheduling algorithm for switching the transmission power level at BTS, based on the output derived from neural network, which is trained with historical data collected from local authorities to learn the population pattern and assign corresponding power levels. The scheduling algorithm with Artificial Neural Network (ANN) gives reduced power consumption, low interference and shortened radiation exposure.
\end{abstract}

Journal of Green Engineering, Vol. 5, 73-84.

doi: 10.13052/jge1904-4720.514

(c) 2015 River Publishers. All rights reserved. 
Keywords: Green Mobile Computing; EM pollution; BTS; Scheduling; PoP.

\section{Introduction}

Wireless technology plays major role in the field of telecommunication where data and voice are frequently exchanged through energy content offered by electromagnetic spectrum. Sophisticated hand on services offered by telecommunication system has increased the number of users. Thus the base stations deployed for managing these load acts as a major artificial source for generating EM radiations. For the major part of the world, to go even a day without coming into contact with any of these devices is impossible. However, most people do not realize that the adverse effects of deterioration of man's living are caused by the electromagnetic radiations that are emitted by all these electronic devices. Though the awareness of this fact is ignored by many, by having a detailed study of this type of radiation and its negative impact and its consequences for human living. The overwhelming research that has been done on this matter provides a substantive evidence and undeniable facts that electromagnetic radiations that has been emitted from high tech devices can be very harmful. Harmful radiations has some other added disadvantages as the link between these radiations and various forms of illness has been revealed through many studies.

As the number of mobile users increased tremendously the energy level of radiations encompassing the environment has also increased and resulted in health hazards. So in order to overcome this, the transmitting power level at BTS is adjusted efficiently based on the number of available user in that environment. A scheduling algorithm accompanied by artificial neural network can be designed for setting the appropriate power level given as output from the neural network trained with data obtained over a period of time. Artificial neural network (ANN) computations unlike von Neumann model do not require separate memory or processing as it operates via the flow of signals through the net connections termed neurons, somewhat akin to biological networks. This artificial neural network is used in predictive modeling, adaptive control, and applications where sufficient datasets are available to train the neurons.Unidirectional Artificial feed forward neural without loops is the first simple ANN which propagates data from input to output via hidden nodes in hidden layer. Reliable wireless communication between the network and end device is achieved using BTS. The end devices like computer, mobile phone, and wireless networks like CDMA, Wi-Fi, and 
GSM. BTS transmit waves that serve a purpose of enabling long distance coverage, but,at the same time pose a threat to the humankind because of its harmful EMF content. This paper deals with dynamically switching of the power levels of BTS according to the requirement. The network is trained to release a power level as an output based on the number of users in that environment. One out of three different power levels to be set for BTS, is estimated as an output from the trained neural network by giving derived parameters as input for analyzing the environment. The work also proposes a scheduling algorithm which takes the output of the ANN and gives the appropriate power level to be set at the corresponding time period.

\section{Related Work}

The scheduling of power levels in the telecommunication networks has been carried out in the various perspectives. One such proposal is made for the WiMax Medium Access Control (MAC) supporting wireless communication utilizes high bandwidth and its QoS requirements vary based on the type of applications employed [1]. MAC does not have a scheduling algorithm to achieve the objective of fairness, QoS and throughput. To satisfy multidimensional objective Scheduling Algorithm along with ANN and Fuzzy is used as it addresses the above mentioned objectives simultaneously. Fairness is achieved as a result, among the users by using the algorithm while keeping priority intact. In addition to this, high channel using up is attained with less computation time. Computer assisted Collaborative Work improvement in effectively combining is considered [2] and for which routers supporting multicast traffic facility is needed. Differentiation between unicast and multicast is not attained with queuing or scheduling. ANN is deployed in power plants for scheduling [3] the needed power to be generated for fulfilling the demands of consumer by prediction using the current load level automatically. Energy reliable wireless communication system has to be designed as increase in energy has led to increased $\mathrm{CO}_{2}$ emission. The number of users belongs to a particular base station is low, then the base station is switched off and the users are served by neighboring stations. Through this concept 20\% [4] of energy consumption is reduced. The drastic growth in telecommunication has resulted in increased health hazards due to high electromagnetic pollution. As a step towards Green Mobile Computing, proposes a model for electromagnetic pollution index and derives the factors influencing the index in order to manage the pollution. The formulation of electromagnetic pollution is made through a packet of pollution (PoP) which helps to devise the methodology 
to reduce EM pollution index in [5]. ANN using back propagation learning is designed to automate the accurate scheduling of power generation plant rating $4 \times 8$ MW [6] depending on the load.

\section{Power Control at BTS Transceiver}

Base Transceiver Station offers reliable wireless connectivity between telecom network and device of end users. Transceiver in BTS is equipped with power amplifier for amplifying the signals before relaying it to the antenna. The maximum transmit power at BTS is determined by the power amplifier rating in BTS. The load of the base station is determined by differences in maximum and present transmission power. The transmitting power level is adjusted based on number of users in the region sufficiently to attain required signal strength. Strong signal strength is needed for end user away from station than the one close to it. Suppose, for a given signal strength if quality of voice is more than sufficient, then the strength can be reduced. Similarly, if there are less number of users in the region than the power level can be reduced considerably. Thus the concept of power control at BTS gives rise to advantages like reduced power consumption, interference, and reduced radiation exposure period. While adjusting the transmission power at BTS the carrier signal strength to interference ratio must be considered for an acceptable power level. Base transceiver station gives $250 \mathrm{nW}$ of power near $1 \mathrm{GHZ}$ and $1 \mu \mathrm{W}$ of power near 12 GHZ [7]. The power generated by BTS is usually constant in a particular frequency band. The model proposed in the system adapts the transmitting power level of transceiver depending on the number of users in particular region of the day.Adapting the transceiver power level helps to reduce unnecessary radiations in the environment where there are only low number of users. The amount of energy in the emitted radiation is also considerably reduced so the ill effects due to power level can be decreased. The radiation exposure for a long time results in some drastic effects like blood pressure, miscarriage, depression, and DNA injury. The proposed model thus reduces the presence of radiations rich in energy by switching the power levels. The constant radiation

exposure for the long term is thus prevented by varying the power levels of radiations in densely populated areas. The mobile users are considerably more in daytime when compared to night time, therefore the power level required during the day is significantly more than the power required to cover the users at night time. So the power level can be reduced during night time as it is sufficient to connect with few mobile users. This has resulted in reduced power consumption and shortened radiation exposure period. The 
conservation of power in turn will reduce the health hazards due to the effects of energy levels in harsh EMF radiations emitted by BTS placed in densely populated areas.

\section{Neural Network Design}

The artificial neural network has input where a number of users are given, hidden for processing and output layer which gives the output power level. The neural network [8] creates the network and train the network using input output values. It uses mean square error and regression models for analyzing the performance of the system. The neural network, thus maps the set of given inputs to the set of target values. Later, the target values can be derived for further processing. This work creates neural network $[9,10]$ to train with the input being number of users and the target being power levels assigned for different range of users. The network is initially trained with datasets having a different range of users as input and correspondingly the target output as power levels collected from local stations for about a week. Once the neural network is trained with analyzed datasets, it is simulated with custom inputs to cross check whether it is working properly. The weighted output of the neural network is the power level, which is to be assigned at BTS for a particular range of users.

The data about the number of users and power level required to satisfy their QoS requirements are collected over a week from local custom base stations. The collected data for every 24 hours is classified as day, afternoon, evening, and night time. The number of users and their power consumption in BTS are analyzed using the collected data. The number of users in day and evening will be more when compared to the number of users at night time. Traffic will also be higher during evening time. The collected dataset over a week about the number of users, time period, and respected power consumptions are given as input to the neural network. After training the network, its performance is measured in terms of mean square error and regression plot as shown in Figures 1 and 2. The power level increase in user and, when the number of user level drops the power level can be reduced which reduces signal strength in that environment. From the Figure 1, it is noticed that the regression value of the plot is very close to 1 , which means the error is minimized to a great extent. The regression value is brought closer to 1 by training the Neural Network with a bigger Dataset. The more the datasets [11] given for training the network, the more accurate the results will be. So the number of possible combinations of verified input and target is given to train the network. 
Hemalatha et al.

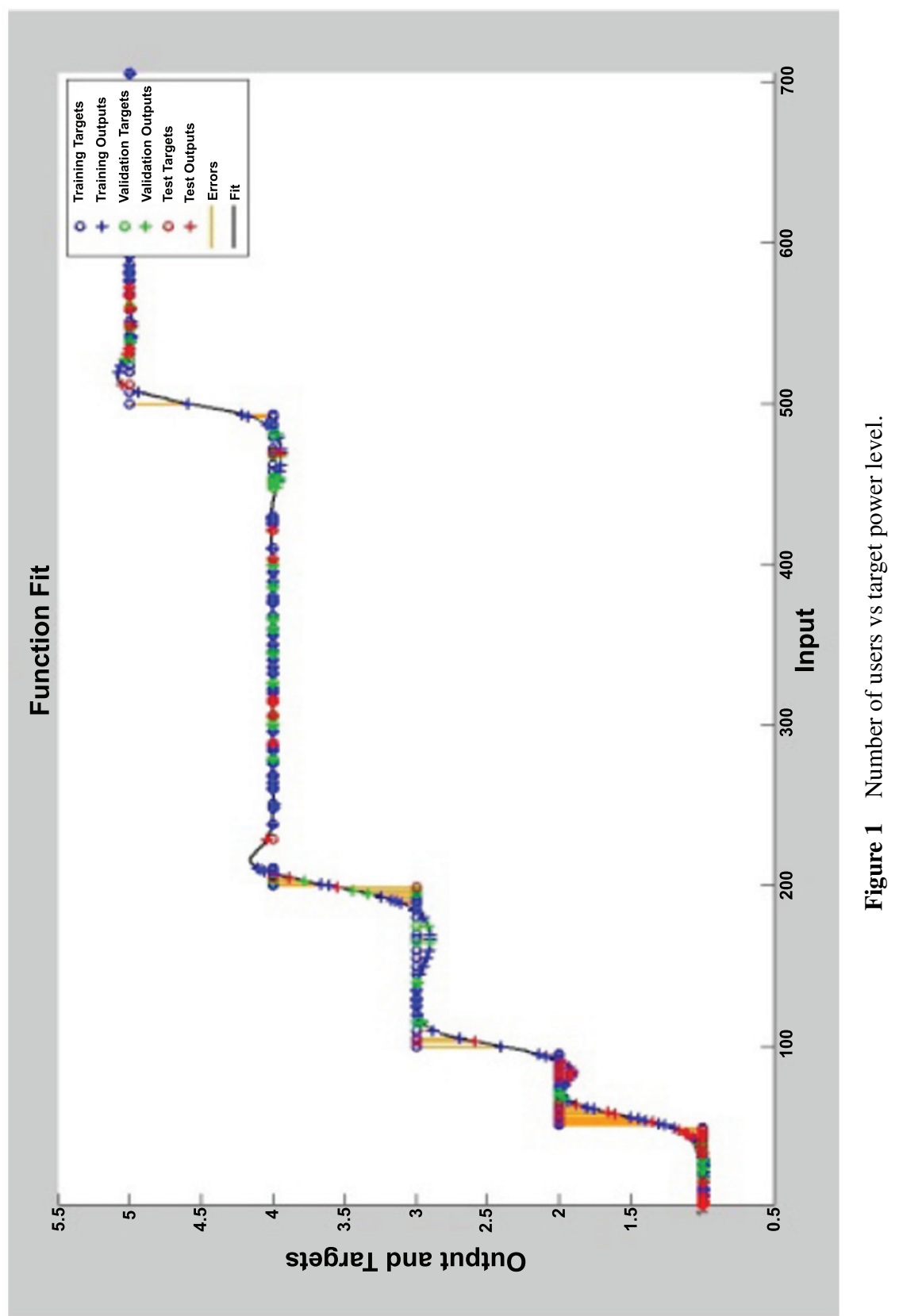




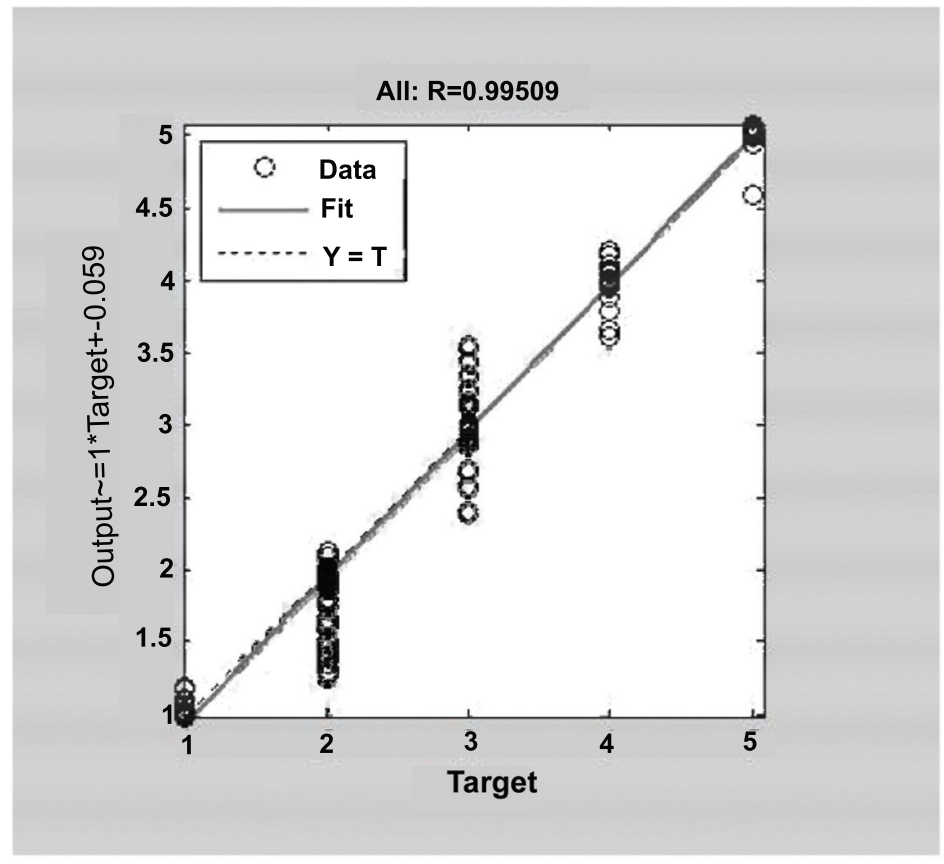

Figure 2 Regression plot.

The dataset given as input for training the neural network is divided into three datasets, as training, validation and testing. The network initially uses training datasets to create a neural mathematical model for the specified input and target.

After creating the model using training data, the neural network uses validation datasets to validate and correct at each step based on the evaluated results. Finally, the test vectors [12] are used to determine the performance of the modeled neural network by evaluating the number of errors. The mean square error can be considerably reduced when more datasets are given for training and the number of iterations required to converge also plays a major role. The Figure 1 shows three different vectors and their correlation with trained values. Figure 2 shows power level assigned to a specific range of user in a town. For e.g. for a range of 1-49 number of users, the power level assigned is 1 . Likewise, there are power levels 2, 3, 4, 5. As the number of users increases the power level is accordingly increased for a particular time of the day. The neural network is now capable of giving power level based on any number of 
user given to it as input. This power level has to be allocated in the appropriate time slot to the BTS.

\section{Scheduling Algorithm for Power Switching}

The scheduling algorithm is basically used to switch the power levels in BTS. The algorithm allocates identified power level in corresponding time slots and switches the BTS to low, medium, or high based on the input provided to ANN. The priority levels are set during day time and increased based on the mobile users in the region due to local functions. The following is the scheduling algorithm designed to allocate the power level.

Step 1: Get the number of users in the cell periodically as input based on some QoS parameter.

Step 2: Create an if-else branch with conditions created by 3 time periods as day, evening, or night. (A)

Step 3: Create a similar loop for packet size and queue length. (B)

Step 4: From the priority levels received in (A) and (B), we create a priority index using if-else branch with three levels namely $\mathrm{L}, \mathrm{M}$, and $\mathrm{H}$

Step 5: The output received is the final priority index showing one among the three available power levels

The priority index points to the power level and packets to be sent to the queue. The appropriate packet to be sent based on the priority index determined using $\mathrm{A}$ and $\mathrm{B}$. Thus the power level is determined based on number of users in the region and BTS is switched to the respective power level for a period of time to transmit the packets.

\section{Implementation}

Along with the scheduling algorithm, this paper also involves an extra parameter known as ILONP (Importance Level of Network Provider). The function of this variable is to combine the functions of all the network providers to one BTS. This variable will decide the priority levels of different network providers. Based on their ILONP values, their request for the frequency band will be met with. This variable combines with the final priority level and gives the decision parameter to the BTS as to whether it should serve the request or 
not. Once the Neural Network is trained and simulated, the model is saved as a network. This model releases an output file whenever it is fed with an input. The error rate for a neural network cannot be more than 0.1. And with this fact, we can easily assume that once we round off the value of power level received from the historical database, it will be the correct value for sure. This power level is taken as Parameter 1. Along with this, three other parameters are left to the network provider to choose from. This will help the Network provider to bring in QoS (Quality of Service) factors at the scheduling level.

Excel file, it will be the correct value for sure. This power level is taken as Parameter 1. Along with this, three other parameters are left to the network provider to choose from. This will help the Network provider to bring in QoS (Quality of Service) factors at the scheduling level.

\section{Results and Discussion}

For the analysis of the prototype, the models were tested with data from the industry. The regression value came close to 0.99 which shows that the model will give near perfect values. The Java code also functioned without any glitches. The prototype, when fully functional, will surely save a lot of power and hopefully save some lives by preventing harmful EMF radiations to spread. This work models, neural network in order to predict the required sum of power level based on the number of users at any given point of time. Back propagation [13] based learning technique gives good result and the amount of data given for training plays an important role. If a sufficient quantity of data is offered to learn, then the network will provide accurate results. The number of hidden nodes impacts the difference in the error between target and estimated value. Also, in our case the scheduling algorithm provides a rounding off function which eliminates the error completely and provides a base for further expansion by providing three parameters for the network providers to decide upon.

\section{Conclusion}

This work constructs soft computing based scheduling technique to resolve any uncertainties present in the outputs which may evolve as errors in the model and interfere with the actual purpose for which the application is deployed. Using ANN to accomplish dynamic switching of power levels, the level of uncertainty is computed and number of inspections were made to 
identify the correlation of output with the given input values. The proposed scheduling algorithm along with neural network reduces the exposure time of the constant high level radiations by switching to low powers where the users are low in number. Hence, the signal strength in the environment and the impact of radiations on living beings can be considerably reduced. The sufficient signal amount provided for transmission also reduces the effect of interference. Therefore the proposed algorithm confirms to green mobile computing by reducing the strength of radiation in the environment through adapting the power level.

\section{References}

[1] D. David Neels Pon Kumar, K. Murugesan, S. Raghavan, and M. Suganthi, "Neural Network based Scheduling Algorithm for WiMAX with improved QoS Constraints", International Conference on Emerging Trends in Electrical and Computer Technology (ICETECT), 2011.

[2] Malika Bourenane, "Energy-Efficient Scheduling Scheme Using Reinforcement Learning in Wireless Ad Hoc Networks", Eighth International Conference on Wireless and Optical Communications Networks (WOCN), 2011.

[3] Meina Song, Xiaosu Zhan and Junde Song "An Efficient Queueing Scheme for Multicast Packet Switching Routers", Eigth International conference on Computer Supported Cooperative Work in Design, 2004.

[4] Prithiviraj Venkatapathy, J Jena, Avadhanulu Jandhyala, "Electromagnetic Pollution Index-A Key Attribute of Green Mobile Communications", Green Technologies Conference (GTC), IEEE, pp. 1-4, 19-20 April 2012.

[5] Prithiviraj, V., Venkatraman, S. B., and Vijayasarathi, R, "Cell zooming for energy efficient wireless cellular network", Journal of Green Engineering, Vol 3, (4), pp. 421-434, 2013.

[6] Mahmoud Moghavvemi, S. S. Yang, and M. A. Kashem, "A Practical Neural Network Approach For Power Generation Automation", Proceedings of EMPD '98 International Conference on Energy Management and Power Delivery, Vol. 1, 1998.

[7] GSM Technical specification, European Telecommunications Standards Institute 1996. 
[8] B.Yegnanarayana, Artificial Neural Networks, PHI Learning Pvt. Ltd, 2004.

[9] Satish Kumar, "Neural Networks: A Classroom Approach", Tata McGraw-Hill, ISBN: 978-0-07048292-02004, 2004.

[10] James. A. Freeman, "Neural Networks: Algorithms, Applications, and Programming Techniques", Pearson Education India, 1991.

[11] S. Geman, E. Bienenstock, and T. Doursat, "Neural networks and the bias/variance dilemma," Neural Comput., vol. 5, pp. 1-58, 1992.

[12] H. Gish, "A probabilistic approach to the understanding and training of neural network classifiers," in Proc. IEEE Int. Conf. Acoustic, Speech,Signal Processing, 1990, pp. 1361-1364.

[13] L. W. Glorfeld, "A methodology for simplification and interpretation of back propagation-based neural networks models," Expert Syst. Applicat., vol. 10, pp. 37-54, 1996.

\section{Biographies}

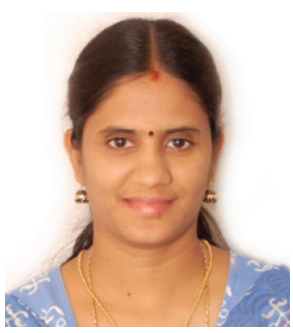

Dr. M. Hemalatha is graduated in Electronics and Communication Engineering, post graduated in Information Technology and Ph.D in Broad band Wireless Communication in the year 1997,2003 and 2012 respectively. She had been associated with SASTRA University, Thanjavur, TN, India, for about 15 years and currently working as Professor in the department of ECE, Narayana Engineering College, Nellore, AP, and India. Having 17+ years of teaching experience, has delivered various technical workshops, lectures and conducted aided projects. Her keen interest in wireless communication and baseband signal processing driven to publish more than 30 articles in the national and international conferences and international journals. 


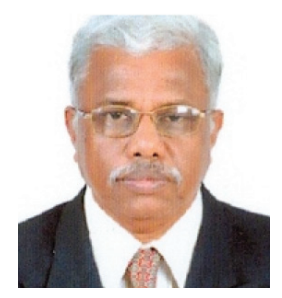

Dr. V. Prithiviraj received M.S degree in Electrical Engineering from IIT Madras., Ph.D. in Electronics and Electrical Communication Engineering from IIT Kharagpur. He is working as Principal Rajalakshmi Institute of Technology from May 2013. He has over 3 decades of teaching experience and 12 years of Research \& Development Experience between the two IITs in the field of RF \& Microwave Engineering. His areas of interest include Broadband and Wireless Communication, Telemedicine, e-Governance and Internet of Things.

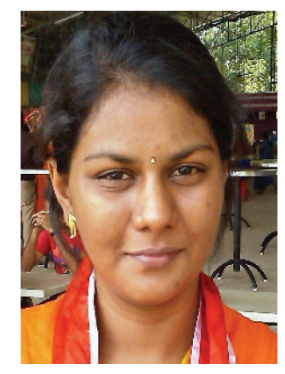

Dr. Jayasri. $\mathbf{R}$ has received B.E(ECE) from Anna University, TN, India and M.Tech(Embedded Systems) from SASTRA University. Having good number of publications in her earlier career, is now working as assistant system engineer in Tata Consultancy Services, Chennai, India. 